This is an electronic reprint of the original article. This reprint may differ from the original in pagination and typographic detail.

Author(s): Seppälä, Otto; Karvonen, Anssi; Rellstab, Christian; Louhi, Katja-Riikka; Jokela, Jukka

Title: $\quad$ Reciprocal interaction matrix reveals complex genetic and dose-dependent specificity among coinfecting parasites

Year: $\quad 2012$

Version:

Please cite the original version:

Seppälä, O., Karvonen, A., Rellstab, C., Louhi, K.-R., \& Jokela, J. (2012). Reciprocal interaction matrix reveals complex genetic and dose-dependent specificity among coinfecting parasites. American Naturalist, 180(3), 306-315.

https://doi.org/10.1086/666985

All material supplied via JYX is protected by copyright and other intellectual property rights, and duplication or sale of all or part of any of the repository collections is not permitted, except that material may be duplicated by you for your research use or educational purposes in electronic or print form. You must obtain permission for any other use. Electronic or print copies may not be offered, whether for sale or otherwise to anyone who is not an authorised user. 


\section{CHICAGO JOURNALS}

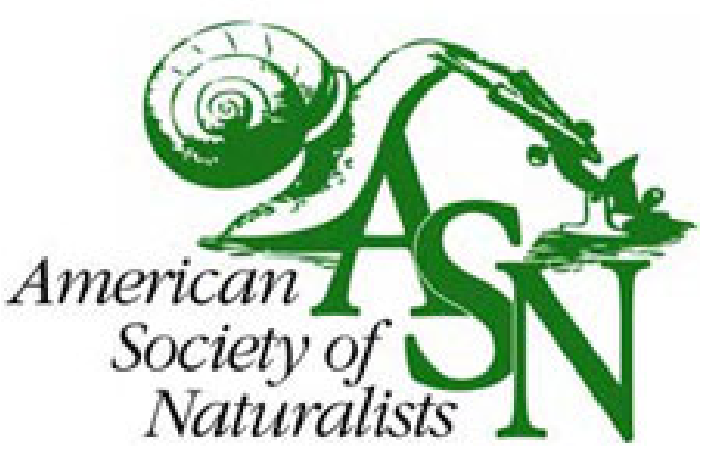

The University of Chicago

Reciprocal Interaction Matrix Reveals Complex Genetic and Dose-Dependent Specificity among Coinfecting Parasites.

Author(s): Otto Seppälä, Anssi Karvonen, Christian Rellstab, Katja-Riikka Louhi, and Jukka Jokela

Source: The American Naturalist, Vol. 180, No. 3 (September 2012), pp. 306-315

Published by: The University of Chicago Press for The American Society of Naturalists

Stable URL: http://www.jstor.org/stable/10.1086/666985

Accessed: 16/12/2015 03:23

Your use of the JSTOR archive indicates your acceptance of the Terms \& Conditions of Use, available at

http://www.jstor.org/page/info/about/policies/terms.jsp

JSTOR is a not-for-profit service that helps scholars, researchers, and students discover, use, and build upon a wide range of content in a trusted digital archive. We use information technology and tools to increase productivity and facilitate new forms of scholarship. For more information about JSTOR, please contact support@jstor.org. 


\title{
Reciprocal Interaction Matrix Reveals Complex Genetic and Dose-Dependent Specificity among Coinfecting Parasites
}

\author{
Otto Seppälä, ${ }^{1,2, \star}$ Anssi Karvonen, ${ }^{3}$ Christian Rellstab, ${ }^{3, \dagger}$ Katja-Riikka Louhi, ${ }^{3,4}$ and Jukka Jokela ${ }^{1,2}$ \\ 1. Eawag, Swiss Federal Institute of Aquatic Science and Technology, 8600 Dübendorf, Switzerland; 2. Swiss Federal Institute of \\ Technology Zürich, Institute of Integrative Biology (IBZ), 8092 Zürich, Switzerland; 3. Centre of Excellence in Evolutionary Research, \\ Department of Biological and Environmental Science, 40014 University of Jyväskylä, Finland; 4. Department of Biological and \\ Environmental Sciences, 00014 University of Helsinki, Finland
}

Submitted March 13, 2012; Accepted May 15, 2012; Electronically published July 19, 2012

Online enhancement: appendix. Dryad data: http://dx.doi.org/10.5061/dryad.8r7s5.

\begin{abstract}
Understanding genetic specificity in factors determining the outcome of host-parasite interactions is especially important as it contributes to parasite epidemiology, virulence, and maintenance of genetic variation. Such specificity, however, is still generally poorly understood. We examined genetic specificity in interactions among coinfecting parasites. In natural populations, individual hosts are often simultaneously infected by multiple parasite species and genotypes that interact. Such interactions could maintain genetic variation in parasite populations if they are genetically specific so that the relative fitness of parasite genotypes varies across host individuals depending on (1) the presence/absence of coinfections and/or (2) the genetic composition of the coinfecting parasite community. We tested these predictions using clones of fish eye flukes Diplostomum pseudospathaceum and Diplostomum gasterostei. We found that interactions among parasites had a strong genetic basis and that this modified genetic variation in infection success of $D$. pseudospathaceum between single and multiple infections as well as across multiply infected host individuals depending on the genetic identity of the coinfecting D. gasterostei. The relative magnitude of these effects, however, depended on the exposure dose, suggesting that ecological factors can modify genetic interactions between parasites.
\end{abstract}

Keywords: host-parasite interactions, multiple infections, Diplostomum pseudospathaceum, Diplostomum gasterostei, Oncorhynchus mykiss, Trematoda.

\section{Introduction}

High within-population genetic variation in host resistance (e.g., Alexander et al. 1993; Grosholz 1994; Kurtz and Sauer 1999; Carius et al. 2001; Koskela et al. 2002; Cotter et al. 2004; Salvaudon et al. 2005; Laine 2007) and parasite infectivity (e.g., Lively 1989; Henter 1995; Carius et al.

\footnotetext{
* Corresponding author; e-mail: otto.seppaelae@eawag.ch.

${ }^{\dagger}$ Present address: Swiss Federal Institute WSL, Zürcherstrasse 111, 8903 Birmensdorf, Switzerland.
}

Am. Nat. 2012. Vol. 180, pp. 306-315. (C) 2012 by The University of Chicago. 0003-0147/2012/18003-53719\$15.00. All rights reserved.

DOI: $10.1086 / 666985$
2001; Kaltz and Shykoff 2002; Laine 2007; Vale and Little 2009) is often observed across a broad range of natural host-parasite interactions both in animal and plant systems. This seems paradoxical because natural selection is expected to erode genetic variation in fitness-related traits by eliminating the least fit alleles from populations (reviewed by Roff 1997). Classically, heterogeneity of the environment (e.g., temperature, resource availability) is considered as an important factor contributing to the maintenance of genetic polymorphism (reviewed by Hedrick 2006), and it has recently been suggested also to be important for maintenance of genetic variation in hostparasite interactions (reviewed by Lazzaro and Little 2009). This is because genetic variation in host resistance often interacts with environmental variation; that is, different host genotypes are most resistant in different environments ( $G \times$ E interaction; e.g., Blanford et al. 2003; Price et al. 2004; Mitchell et al. 2005; Seppälä and Jokela 2010). Thus, pathogen-mediated selection may favor different host genotypes under different environmental conditions, which promotes maintenance of genetic polymorphism in host populations.

The role of environmental variation in maintaining genetic variation in parasites is, however, still generally poorly understood (but see Fels and Kaltz 2006; Laine 2007; Vale and Little 2009; Bryner and Rigling 2011). Since hosts represent the parasites' primary environment, a potentially important environmental factor modifying their fitness is the presence of coinfecting parasites. In fact, individual hosts are often simultaneously infected by multiple parasite species (e.g., Holmes and Price 1986; Valtonen et al. 2001) and genotypes (reviewed by Read and Taylor 2001) that interact (e.g., Lello et al. 2004; de Roode et al. 2005; Koskella et al. 2006; Staves and Knell 2010; Laine 2011). Furthermore, because the genetic composition of the coinfecting parasite community is typically variable across multiply infected host individuals (i.e., dif- 
ferent hosts are infected by different parasite species and genotypes), we propose that the outcomes of interactions among parasites could show high variation, potentially contributing to the maintenance of genetic variation. However, this is possible only if these interactions are genetically specific so that the relative fitness of parasite genotypes varies depending on the presence or absence of coinfections and/or the genetic composition of the coinfecting parasite community.

We tested these hypotheses using Diplostomum (Trematoda) eye flukes of fish. Diplostomum parasites are common in several freshwater fishes, and multiple-species (Valtonen and Gibson 1997; Rellstab et al. 2011) and multiple-genotype (Rauch et al. 2005) infections are the rule in nature. In this study, we used D. pseudospathaceum (called Diplostomum spathaceum in our earlier studies [Seppälä et al. 2007, 2009] but revealed to be D. pseudospathaceum using genetic analysis [Louhi et al. 2010]) and $D$. gasterostei (synonym Diplostomum baeri). We estimated variation in parasite fitness by assaying their infection success, which is an important fitness related trait. Natural populations of these parasite species show high amonggenotype/isolate variation in their infectivity to fish (Seppälä et al. 2007, 2009; Rauch et al. 2008; Voutilainen et al. 2010; Karvonen et al. 2012). Furthermore, these parasites interact in fish so that the infection success of $D$. pseudospathaceum is modified in coexposures (Seppälä et al. 2009). Interestingly, the effect of interaction is highly variable suggesting a complex genetic or ecological interaction between these parasite species (Seppälä et al. 2009).

In this study, we examined the potential role of coinfections in maintaining genetic variation in parasites by testing whether the observed variation in the outcome of coinfections (Seppälä et al. 2009) has a genetic basis. We measured the infection success of D. pseudospathaceum genotypes in single-genotype exposures and in coexposures with different $D$. gasterostei isolates using a reciprocal infection matrix (we did not measure the infection success of $D$. gasterostei because it was not affected by coexposure with D. pseudospathaceum in our earlier study; Seppälä et al. 2009). This experiment reveals whether the outcome of coexposure is determined by genetic identity of $D$. pseudospathaceum, D. gasterostei, or both ( $\mathrm{G} \times \mathrm{G}$ interaction), which is important in determining under which conditions coinfections could maintain genetic variation. Interactions between parasites can promote maintenance of genetic polymorphism if coinfections change the relative fitness of parasite genotypes (1) between single and multiple infections and/or (2) across different multiply infected hosts depending on the genetic identity of the coinfecting parasite. Furthermore, we examined the effect of exposure dose, which is largely determined by ecological factors, on the outcome of interactions among parasites. This is rel- evant as wild fish are typically exposed to variable doses of parasite transmission stages. We predicted that dose effects may modify the nature of genetic specificity of the interactions through induced host immune responses.

\section{Material and Methods \\ Study Organisms}

Trematodes of the genus Diplostomum have three-host life cycles (see Williams 1966; Chappell et al. 1994). Parasites mature in the intestine of fish-eating birds and reproduce sexually. Their eggs are released to water with birds' feces and hatch into free-swimming miracidia larvae. Miracidia of Diplostomum pseudospathaceum infect mainly Lymnaea stagnalis snails (Louhi et al. 2010), whereas Diplostomum gasterostei infects mainly Radix balthica and Myxas glutinosa snails (Karvonen et al. 2006). Parasites penetrate into snail gonads, where they develop into sporocysts and multiply asexually. Sporocysts produce thousands of freeswimming cercariae larvae that leave the snail and seek a fish. Because multiplication of Diplostomum parasites in snails is asexual, all cercariae originating from a single miracidial infection represent one genetically identical clone. Cercariae of both parasite species infect fish by penetrating the gills and skin and migrating to the eyes, where they develop into metacercariae. Metacercariae of D. pseudospathaceum locate themselves in the lenses whereas $D$. gasterostei locates itself in the vitreous body (Williams 1966; Karvonen et al. 2006), which could be one potential reason why only $D$. pseudospathaceum was affected by coexposure in our previous study (i.e., only D. pseudospathaceum migrates through the organ inhabited by the other species; see Seppälä et al. 2009). For successful completion of the life cycle, an infected fish has to be eaten by a piscivorous bird.

We collected naturally infected snail hosts of the parasites (D. pseudospathaceum: Lymnaea stagnalis; D. gasterostei: Radix balthica) from Lake Konnevesi $\left(62^{\circ} 37^{\prime} \mathrm{N}\right.$, $26^{\circ} 21^{\prime} \mathrm{E}$ ) in Finland. We separated snails infected with $D$. pseudospathaceum and D. gasterostei from snails infected with other parasite species by observing the morphology and behavior of released cercariae (see Karvonen et al. 2006). We used only D. pseudospathaceum cercariae from snails infected with one parasite genotype. We verified this by genotyping 20 cercariae from each snail using polymorphic microsatellite markers (Reusch et al. 2004) as described in Louhi et al. (2010). All clones derived from different snail individuals represented unique multilocus genotypes. We did not genotype D. gasterostei cercariae because microsatellite markers are not available for this species. Therefore, it is possible that some $R$. balthica snails released more than one parasite genotype. However, since 
all infections in the snails originated from sexually produced miracidia, we know that D. gasterostei cercariae derived from different snail individuals were genetically distinct parasite isolates and therefore suitable for testing the responses of $D$. pseudospathaceum genotypes to genetically varying coinfections. Furthermore, the possible "noise" in the data caused by multiple-genotype infections in D. gasterostei infected snails would lead to a conservative error. This is because if multiple infections were common, the probability of detecting differences in the outcome of interaction among parasites would be reduced because among-isolate variance in D. gasterostei would be confounded with within-isolate variance (see Luijckx et al. 2011). Before the experiment (see below), we fed all the snails ad lib. with fresh lettuce for 4 days to minimize the possible variation in quality of released cercariae that could arise from differences in the snails' physiological condition (see Seppälä et al. 2008).

We used juvenile $(0+$ years old) rainbow trout $(\mathrm{On}$ corhynchus mykiss) as a fish host in the experiment. We obtained the fish from a commercial fish farm where they had been reared in indoor tanks supplied with groundwater. This ensured that the fish had no previous experience of eye flukes or other helminth parasites.

\section{Experimental Design}

To produce parasite cercariae for the experiment, we placed six infected L. stagnalis and four infected $R$. balthica snails individually in glass jars containing $2 \mathrm{dL}$ of water $\left(17^{\circ} \mathrm{C}\right)$ and allowed them to shed cercariae for $6 \mathrm{~h}$. We estimated the density of cercariae in each jar from 10 1mL samples.

In the experiment, we measured the infection success of each $D$. pseudospathaceum genotype to fish in singlegenotype exposures and in mixed-species coexposures by testing each genotype against each of the four D. gasterostei isolates separately (fig. 1). We used two exposure doses (100 and 50 cercariae of each parasite species per fish) in each exposure treatment to control for possible dose effects (fig. 1). This is important because exposure dose may, for example, determine the strength and type of host immune responses (see Bancroft et al. 2001; Bleay et al. 2007). We did not use a lower exposure dose than 50 cercariae of each parasite species per fish because the statistical power in such a treatment would have been low due to low infection levels (see analyses below).

In each treatment combination, we exposed 10 randomly selected fish to infection by placing the fish individually into containers with $0.5 \mathrm{~L}$ of water and cercariae for $30 \mathrm{~min}$ at $17^{\circ} \mathrm{C}$. We distributed the cercariae into the containers using a microtiter pipette. We determined the volume of cercariae suspension needed based on its esti-
Single-genotype exposures

D. pseudospathaceum genotype

\begin{tabular}{|c|c|c|c|c|c|c|}
\hline \multirow{3}{*}{$\begin{array}{l}\mathscr{~} \\
\mathscr{W} \\
\text { О }\end{array}$} & 1 & 2 & 3 & 4 & 5 & 6 \\
\hline & 10 & 10 & 10 & 10 & 8 & 10 \\
\hline & 10 & 10 & 10 & 10 & 10 & 10 \\
\hline
\end{tabular}

Mixed-species coexposures

D. pseudospathaceum genotype

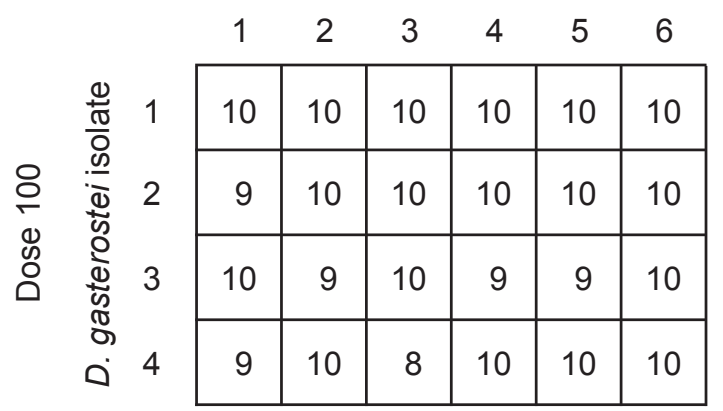

\begin{tabular}{|c|c|c|c|c|c|c|c|}
\hline \multirow{4}{*}{ 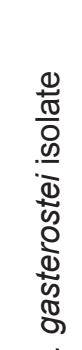 } & & 1 & 2 & 3 & 4 & 5 & 6 \\
\hline & 1 & 10 & 10 & 10 & 10 & 10 & 10 \\
\hline & 2 & 10 & 10 & 10 & 10 & 10 & 10 \\
\hline & 3 & 10 & 10 & 10 & 10 & 8 & 10 \\
\hline & 4 & 10 & 10 & 9 & 10 & 10 & 10 \\
\hline
\end{tabular}

Figure 1: Experimental design used in the study showing different exposure treatments as well as replication (number of fish used in the analyses) in each treatment combination.

mated cercariae density (see above) and the used exposure dose. After the exposure, we transferred the fish to four 1400-L tanks, where we maintained the fish from different treatments separately in cages $(40 \mathrm{~cm} \times 40 \mathrm{~cm} \times 40 \mathrm{~cm})$. We killed the fish $48 \mathrm{~h}$ postexposure (parasites establish in the eyes within $24 \mathrm{~h}$ from exposure; Whyte et al. 1991) with an overdose of $0.01 \%$ MS 222 (Sigma Chemical, St. Louis). We measured the length $( \pm 1 \mathrm{~mm})$ of the fish and counted the number of $D$. pseudospathaceum parasites in fish eyes after dissecting them. We did not measure infection success of $D$. gasterostei because it was not affected by coexposure in our earlier experiment (Seppälä et al. 2009). Twelve fish died before the dissection or we could 
not determine the parasite numbers in their eyes. We excluded these fish from the data (see fig. 1).

\section{Statistical Analyses}

To assess whether $D$. pseudospathaceum genotypes differed in their infectivity when not interacting with coinfecting parasites, we analyzed the variation in parasite infection success in single-genotype exposures using ANCOVA. In the analysis, we used the proportion of cercariae successfully infecting fish as a response variable, $D$. pseudospathaceum genotype $\left(G_{\mathrm{p}}, 6\right.$ genotypes) as a random factor, exposure dose (D, 2 doses) as a fixed factor, and fish length as a covariate. Since the interaction term between genetic identity of D. pseudospathaceum and exposure dose $\left(G_{\mathrm{P}} \times \mathrm{D}\right.$ interaction) approached statistical significance in this model (table 1; $P=.066$ ), we further analyzed the variation in infection success separately for different exposure doses. We did this to examine whether parasite infectivity showed genetic variation in both exposure doses.

To analyze variation in interactions among coinfecting parasites, we first calculated the relative infection success of $D$. pseudospathaceum parasites in coexposures when compared to their infectivity in single-genotype exposures. In other words, we scaled parasite infection success in coexposures to their infectivity in single-genotype exposures to get a response variable that reflects the change in parasite performance between the exposure types. To do this, we first calculated the expected infection success of D. pseudospathaceum for each fish individual in coexposure treatments using the ANCOVA model for parasite infection success in single-genotype exposures (see above). We used this model rather than the average infectivity of each parasite genotype in single-genotype exposures because fish length affected parasite infection success (table 1). We then calculated the relative infection success of parasites in coexposures by dividing the observed infection success (proportion of cercariae successfully infecting fish) in each fish individual by its expected infection success. This variable shows how much parasite performance increased (values larger than 1) or decreased (values between 0 and 1) in coexposures when compared to single-genotype exposures. We log transformed these values to transform the data from relative to additive for the analyses. We analyzed the variation in relative infection success in coexposures using an ANCOVA with genetic identity of $D$. pseudospathaceum $\left(\mathrm{G}_{\mathrm{p}}, 6\right.$ genotypes) and D. gasterostei $\left(\mathrm{G}_{\mathrm{G}}, 4\right.$ isolates) as random factors, exposure dose (D, 2 doses) as a fixed factor, and fish length as a covariate. When a statistically significant interaction between genetic identity of D. pseudospathaceum and exposure dose (i.e., $\mathrm{G}_{\mathrm{P}} \times \mathrm{D}$ and/ or $G_{P} \times G_{G} \times D$ interaction) was observed, we further analyzed the variation in relative infection success of the
Table 1: ANCOVA for the infection success of Diplostomum pseudospathaceum in single-genotype exposures

\begin{tabular}{lrrrc}
\hline Source & df & MS & \multicolumn{1}{c}{$F$} & $P$ \\
\hline D. pseudospathaceum $\left(\mathrm{G}_{\mathrm{P}}\right)$ & 5 & .087 & $1.527^{\mathrm{a}}$ & .328 \\
Dose $(\mathrm{D})$ & 1 & .004 & $.066^{\mathrm{a}}$ & .808 \\
Fish length & 1 & .113 & 4.243 & .042 \\
$\mathrm{G}_{\mathrm{p}} \times \mathrm{D}$ & 5 & .057 & 2.139 & .066 \\
Error & 105 & .027 & &
\end{tabular}

Note: Factors are the genetic identity of $D$. pseudospathaceum (6 genotypes, random factor), exposure dose (2 doses, fixed factor), and fish length (covariate).

a $\left(G_{p} \times D\right)$ as the error term.

parasites in coexposures separately for different exposure doses. We did this to examine whether genetic specificity (i.e., main effect of $\mathrm{G}_{\mathrm{P}}$ and/or $\mathrm{G}_{\mathrm{P}} \times \mathrm{G}_{\mathrm{G}}$ interaction) among interacting parasites was observed in both exposure doses.

The above analyses reveal the genetic specificity and its dose dependence in the interactions between the parasites, but do not estimate whether these interactions significantly modify the genetic variation in infectivity of $D$. pseudospathaceum among exposure treatments. To examine this, we first analyzed the variation in the absolute infection success of $D$. pseudospathaceum genotypes in different coexposure treatments (i.e., we did not scale parasite infection success to their infectivity in single-genotype exposures) using similar ANCOVA models as above. When general differences in the infection success of parasite genotypes (main effect of $\mathrm{G}_{\mathrm{P}}$ ) was observed in coexposures, we examined whether differences in their infectivity in single-genotype exposures with the same exposure dose significantly predicted this variation. We did this using a linear regression with the mean infection success of each genotype in mixed-species coexposures (averaged across D. gasterostei isolates, fish length-adjusted) as a dependent variable and the mean infectivity of the same genotypes in their respective single-genotype exposures (fish lengthadjusted) as an independent variable. This analysis reveals whether the genetic variation in parasite infection success is consistent or modified between single exposures and multiple exposures. Accordingly, the interaction terms between the genetic identities of coinfecting parasites $\left(G_{P} \times\right.$ $\mathrm{G}_{\mathrm{G}}$ interaction) in the ANCOVAs reveal whether or not the genetic variation in parasite infection success varies across different multiply infected host individuals. Assumptions of the all analyses were fulfilled, and we performed them using IBM SPSS 19.0 (SPSS, Chicago) software.

\section{Results}

Our analysis did not show a significant main effect of parasite genotype in determining the infectivity of $D$. pseu- 

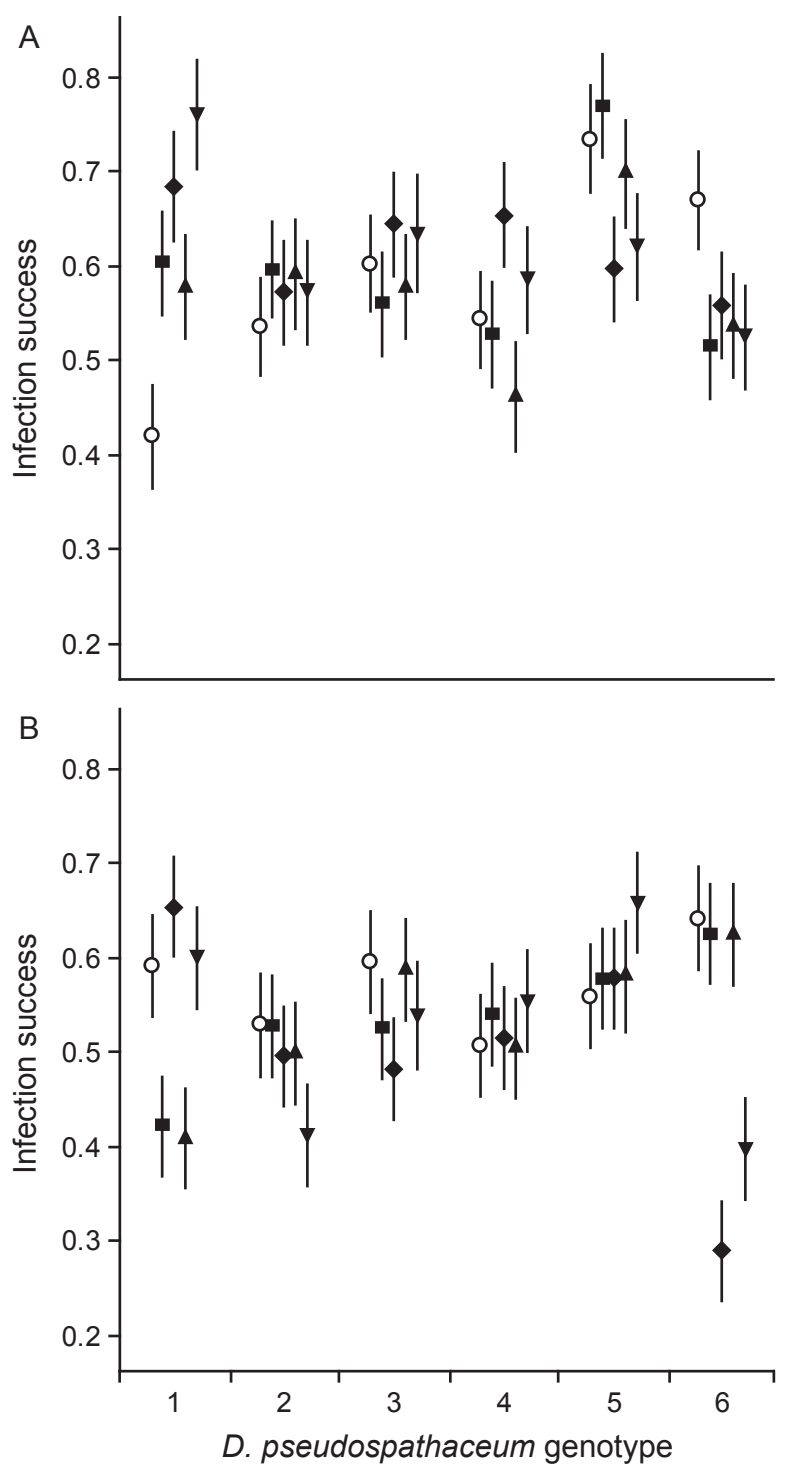

Figure 2: Infection success (fish length-adjusted mean \pm SE) of six Diplostomum pseudospathaceum genotypes in single-genotype exposures (open symbols) and in coexposures with four different $D i-$ plostomum gasterostei isolates (black symbols) measured as a proportion of parasites that successfully infected fish eyes. Each black symbol represents one $D$. pseudospathaceum genotype when tested against one $D$. gasterostei isolate (each symbol indicates one isolate). $A$ and $B$ refer to the two exposure doses (100 and 50 cercariae of each parasite species per fish, respectively) used in the experiment.

dospathaceum in single-genotype exposures (table 1). However, the interaction term between parasite genotype and exposure dose was close to statistical significance (table 1), which suggests that genetic variation in parasite infectivity may vary across exposure doses. Separate statistical models for each dose treatment revealed that this was be- cause infectivity of $D$. pseudospathaceum genotypes differed in the high-exposure dose treatment (100 cercariae per fish; ANCOVA, $F_{5,51}=4.410, P=.002$; table A1, available online; fig. $2 A$ ) but not in the low-exposure dose treatment (50 cercariae per fish; ANCOVA, $F_{5,53}=0.818$, $P=.542$; table A1; fig. $2 B)$.

In coexposures, D. pseudospathaceum showed genetic specificity in its responses to the presence of D. gasterostei. In other words, the relative infection success of $D$. pseudospathaceum in coexposures when compared to singlegenotype exposures varied across different genotypes, as well as across different combinations of D. pseudospathaceum genotype and D. gasterostei isolate (table 2; fig. 2). These effects, however, depended on exposure dose as indicated by significant $G_{P} \times D$ and $G_{P} \times G_{G} \times D$ interactions (table 2). When the dose treatments were analyzed separately, in the high-exposure dose, D. pseudospathaceum genotypes showed general differences in their responses to coexposure that were independent of the genetic identity of the coinfecting partner (main effect of $\mathrm{G}_{\mathrm{p}}$ : ANCOVA, $F_{5,15}=17.570, P<.001$; table A2, available online; fig. $2 A$ ). In the low-exposure dose treatment, $D$. pseudospathaceum genotypes did not show general differences in their responses to coexposures, but the outcome of interaction depended on the genetic identity of the both interacting parasite species $\left(\mathrm{G}_{\mathrm{P}} \times \mathrm{G}_{\mathrm{G}}\right.$ interaction: ANCOVA, $F_{15,212}=4.232, P<.001$; table A2; fig. $\left.2 B\right)$.

The observed genetic specificity in the interactions among coinfecting parasites modified the absolute infection success of $D$. pseudospathaceum genotypes (table 3; fig. 2). However, also this effect depended on the exposure dose as indicated by a significant $G_{P} \times G_{G} \times D$ interaction

Table 2: ANCOVA for the relative infection success of Diplostomum pseudospathaceum in coexposures with Diplostomum gasterostei when compared to the infectivity in single-genotype exposures

\begin{tabular}{lrrrc}
\hline Source & df & MS & $F$ & $P$ \\
\hline D. pseudospathaceum $\left(\mathrm{G}_{\mathrm{P}}\right)$ & 5 & .325 & $1.211^{\mathrm{a}}$ & .411 \\
D. gasterostei $\left(\mathrm{G}_{\mathrm{G}}\right)$ & 3 & .011 & $.180^{\mathrm{b}}$ & .904 \\
Dose $(\mathrm{D})$ & 1 & .410 & $1.666^{\mathrm{c}}$ & .259 \\
Fish length & 1 & .104 & 5.513 & .019 \\
$\mathrm{G}_{\mathrm{P}} \times \mathrm{D}$ & 5 & .251 & $5.417^{\mathrm{d}}$ & .005 \\
$\mathrm{G}_{\mathrm{G}} \times \mathrm{D}$ & 3 & .041 & $.887^{\mathrm{d}}$ & .470 \\
$\mathrm{G}_{\mathrm{P}} \times \mathrm{G}_{\mathrm{G}}$ & 15 & .064 & $1.373^{\mathrm{d}}$ & .273 \\
$\mathrm{G}_{\mathrm{P}} \times \mathrm{G}_{\mathrm{G}} \times \mathrm{D}$ & 15 & .046 & 2.459 & .002 \\
Error & 421 & .019 & & \\
\hline
\end{tabular}

Note: Factors are the genetic identity of $D$. pseudospathaceum (6 genotypes, random factor) and D. gasterostei (4 isolates, random factor), exposure dose (2 doses, fixed factor), and fish length (covariate).

a $\left(G_{p} \times D\right)+\left(G_{P} \times G_{G}\right)-\left(G_{P} \times G_{G} \times D\right)$ as the error term.

b $\left(G_{G} \times D\right)+\left(G_{p} \times G_{G}\right)-\left(G_{P} \times G_{G} \times D\right)$ as the error term.

c $\left(G_{P} \times D\right)+\left(G_{G} \times D\right)-\left(G_{P} \times G_{G} \times D\right)$ as the error term.

d $\left(G_{P} \times G_{G} \times D\right)$ as the error term. 
Table 3: ANCOVA for the absolute infection success of Diplostomum pseudospathaceum in coexposures with Diplostomum gasterostei

\begin{tabular}{lrrrr}
\hline Source & $\mathrm{df}$ & $\mathrm{MS}$ & \multicolumn{1}{c}{$F$} & \multicolumn{1}{c}{$P$} \\
\hline D. pseudospathaceum $\left(\mathrm{G}_{\mathrm{P}}\right)$ & 5 & .153 & $2.846^{\mathrm{a}}$ & .178 \\
D. gasterostei $\left(\mathrm{G}_{\mathrm{G}}\right)$ & 3 & .007 & $.132^{\mathrm{b}}$ & .935 \\
Dose $(\mathrm{D})$ & 1 & .636 & $175.746^{\mathrm{c}}$ & .901 \\
Fish length & 1 & .978 & 33.683 & $<.001$ \\
$\mathrm{G}_{\mathrm{P}} \times \mathrm{D}$ & 5 & .028 & $.523^{\mathrm{d}}$ & .756 \\
$\mathrm{G}_{\mathrm{G}} \times \mathrm{D}$ & 3 & .030 & $.544^{\mathrm{d}}$ & .659 \\
$\mathrm{G}_{\mathrm{P}} \times \mathrm{G}_{\mathrm{G}}$ & 15 & .080 & $1.465^{\mathrm{d}}$ & .234 \\
$\mathrm{G}_{\mathrm{P}} \times \mathrm{G}_{\mathrm{G}} \times \mathrm{D}$ & 15 & .055 & 1.878 & .024 \\
Error & 421 & .029 & & \\
\hline
\end{tabular}

Note: Factors are the genetic identity of D. pseudospathaceum (6 genotypes, random factor) and D. gasterostei (4 isolates, random factor), exposure dose (2 doses, fixed factor), and fish length (covariate).

${ }^{a}\left(G_{\mathrm{P}} \times D\right)+\left(G_{\mathrm{P}} \times G_{G}\right)-\left(G_{\mathrm{P}} \times G_{G} \times D\right)$ as the error term.

${ }^{b}\left(G_{G} \times D\right)+\left(G_{p} \times G_{G}\right)-\left(G_{p} \times G_{G} \times D\right)$ as the error term.

c $\left(G_{\mathrm{P}} \times \mathrm{D}\right)+\left(\mathrm{G}_{\mathrm{G}} \times \mathrm{D}\right)-\left(\mathrm{G}_{\mathrm{p}} \times \mathrm{G}_{\mathrm{G}} \times \mathrm{D}\right)$ as the error term.

${ }^{d}\left(G_{P} \times G_{G} \times D\right)$ as the error term.

(table 3). When the dose treatments were analyzed separately, in the high-exposure dose, D. pseudospathaceum genotypes showed general differences in their infection success in coexposures that were independent of the genetic identity of coinfecting $D$. gasterostei isolate (main effect of $\mathrm{G}_{\mathrm{P}}$ : ANCOVA, $F_{5,15}=3.082, P=.041$; table A3, available online; fig. $2 A$ ). Infectivity of the same genotypes in single-genotype exposures, however, did not explain the observed variation (linear regression, $R^{2}=0.003, F_{1,4}=$ $0.011, P=.920$; fig. 3 ). In the low-exposure dose treatment, infection success of the parasites in coexposures varied across different combinations of $D$. pseudospathaceum genotype and D. gasterostei isolate $\left(\mathrm{G}_{\mathrm{P}} \times \mathrm{G}_{\mathrm{G}}\right.$ interaction: ANCOVA, $F_{15,212}=3.466, P<.001$; table A3; fig. $2 B)$.

\section{Discussion}

Natural selection is expected to erode genetic variation in fitness related traits by eliminating the least fit alleles from populations (reviewed by Roff 1997). However, traits that determine the outcome of host-parasite interactions often show high genetic variation in nature (e.g., host resistance: Alexander et al. 1993; Grosholz 1994; Kurtz and Sauer 1999; Carius et al. 2001; Koskela et al. 2002; Cotter et al. 2004; Salvaudon et al. 2005; Laine 2007; parasite infectivity: Lively 1989; Henter 1995; Carius et al. 2001; Kaltz and Shykoff 2002; Laine 2007; Vale and Little 2009). Recently, heterogeneity of the environment has been acknowledged as a potential mechanism maintaining genetic polymorphism in host resistance (e.g., Blanford et al. 2003; Price et al. 2004; Mitchell et al. 2005; Seppälä and Jokela 2010), but its importance in maintaining genetic variation in par- asites is still poorly understood (but see Fels and Kaltz 2006; Laine 2007; Vale and Little 2009; Bryner and Rigling 2011). In this study, we examined whether coinfecting parasites could introduce variation to the relative fitness of parasite genotypes through genetically specific interactions that modify infection success. By conducting experimental single-genotype exposures and mixed-species coexposures using Diplostomum pseudospathaceum and Diplostomum gasterostei eye flukes of fish, we found that interactions between these species were genetically specific and that they introduced significant variation to the infection success of D. pseudospathaceum. Furthermore, the observed effects depended on the exposure dose, which suggests that the ecological context of the actual exposure event can modify the genetic interactions determining parasite infection success.

In the high-exposure dose treatment (100 cercariae of each parasite species per fish), responses of D. pseudospathaceum to coexposures were specific to each parasite genotype, but did not depend on the genetic identity of the coinfecting D. gasterostei. This modified the absolute infection success of $D$. pseudospathaceum so that the infection success of parasite genotypes when compared to

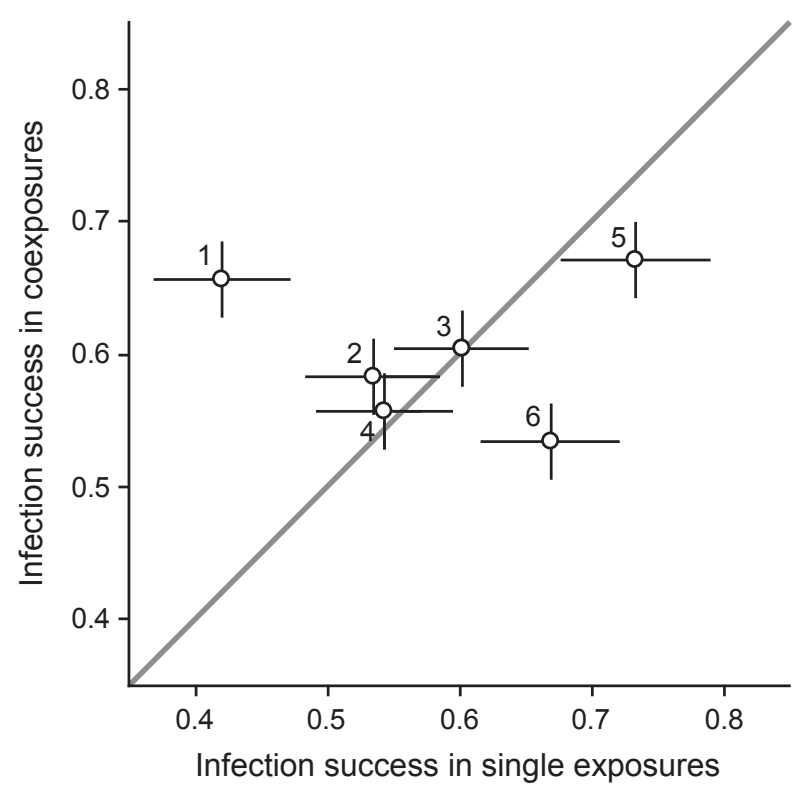

Figure 3: Infection success (fish length-adjusted mean \pm SE) of six Diplostomum pseudospathaceum genotypes in single-genotype exposures and in coexposures with Diplostomum gasterostei (averaged across different isolates) in the high-exposure dose treatment (100 cercariae of each parasite species per fish) measured as a proportion of parasites that successfully infected fish eyes. Gray line indicates equal infection success in both treatments, and numbers next to the error bars refer to the identity of different $D$. pseudospathaceum genotypes as in figure 2 . 
each other changed between singly and multiply infected host individuals. In the low-exposure dose treatment (50 cercariae of each parasite species per fish), the outcome of interaction depended on the genetic identity of the both parasite species. This modified the genetic variation in parasite infectivity across different multiply infected hosts. This kind of context dependence in infection success could erode most of the expected direct relationship between parasite genotype and fitness. Therefore, as variable environmental factors in general (see Gillespie and Turelli 1989; Schlichting and Pigliucci 1998), interactions among coinfecting parasites should promote maintenance of genetic variation by favoring different alleles of the parasites' genes depending on the presence/absence of coinfecting parasites and the genetic composition of the coinfecting parasite community within a host.

In studies examining interactions among coinfecting parasites, variation in exposure dose between single exposures and coexposures needs to be considered as a potential confounding factor as it is inevitable that either the total number of parasites or the relative dose of interacting partners differs between the treatments (the former is true in this study). This is because if parasite performance is dose dependent, differences between exposure treatments could simply reflect differences in the number of parasites the hosts are exposed to (Taylor et al. 1997). In our study, such dose effects could possibly confound the observed changes in parasites' infection success between singlegenotype exposures and mixed-species coexposures in the high-exposure dose treatment. We, however, think this is unlikely because the infection success of Diplostomum cercariae in single-species exposures is known to be largely independent of the exposure dose within the range of doses used here (e.g., Brassard et al. 1982; Stables and Chappell 1986; Whyte et al. 1991). This is also the case for $D$. pseudospathaceum (Karvonen et al. 2003; this study) and D. gasterostei (A. Karvonen et al., personal observations, 2004). Verification of this, however, would have required additional treatments with higher-exposure doses in single-genotype exposures to be included in the experimental design. In the low-exposure dose treatment, the observed dependence of variation in the absolute infection success of $D$. pseudospathaceum genotypes from the genetic identity of coinfecting D. gasterostei cannot be confounded by potential dose effects because the exposure doses (both the total number of cercariae and the relative number of interacting partners) were the same across different combinations of coinfecting parasites. This allows us to interpret those results as interactions among parasites.

It is also important to note that each of the parasite genotypes/isolates used in this study originated from an individual snail host. Therefore, variation in the physiological condition of the snails could have affected the qual- ity of released cercariae larvae and thus potentially modified their infection success. In fact, in our study system, starvation of snails is known to reduce parasite's reproductive rate and life span of produced cercariae (Seppälä et al. 2008), which is most likely due to limited amount of resources available for the parasite. As replicating each parasite clone across several snail individuals is impossible in our study system (clonal reproduction takes place only within snails, and larval stages infecting snails [miracidia] are produced through sexual reproduction), we aimed to reduce the possible variation in quality of the used cercariae due to effects of host condition by feeding all the snails ad lib. with fresh lettuce for 4 days before the experiment.

The reasons for the observed genetic specificity and its dependence on the exposure dose in interactions between D. pseudospathaceum and D. gasterostei are not fully understood. In general, interactions among coinfecting parasites can take place through direct competition (Patrick 1991; Selva et al. 2009; Staves and Knell 2010) and/or indirectly through host defenses (Adams et al. 1989; Råberg et al. 2006; Ezenwa et al. 2010; Laine 2011). In most study systems, separation between these mechanisms is difficult. In our system, however, interaction through direct competition is unlikely. This is because $D$. pseudospathaceum and D. gasterostei infect different parts of fish eyes (eye lenses and vitreous bodies, respectively), when competition for space and resources in the eyes should be weak or negligible. Furthermore, possible resource competition should be most intense during parasite growth and development, not during establishment, studied in this experiment. Similarly, although we cannot fully exclude the possibility that Diplostomum parasites interact directly while invading the fish and/or migrating to the eyes, such effects are also likely to be weak because the parasites can use the whole surface area of the fish for the invasion (Erasmus 1959; Chappell et al. 1994) reducing the probability of contact during establishment. Lack of direct competition among these parasite species is also supported by our previous study in which the effect of coexposure on the infection success of $D$. pseudospathaceum was not explained by the performance of the coinfecting D. gasterostei isolate as could have been predicted if the highly infectious isolates of one species were better in inhibiting the infection success of the other (Seppälä et al. 2009).

In this study, we used fish that had no previous experience of Diplostomum eye flukes. Furthermore, because we examined the fish $48 \mathrm{~h}$ postexposure, the fish did not develop acquired immune defense against the infections during the experiment (development of acquired immunity takes 3-4 weeks depending on water temperature; Aaltonen et al. 1994; Chappell et al. 1994). Thus, the observed interactions among parasites are likely to be mediated by the 
innate immune defense of fish. This is possible because recent studies, including also Diplostomum-fish interaction, have revealed that parasite-genotype-specific host responses occur in the innate immune defense (Schmid-Hempel 1999; Carius et al. 2001; Rauch et al. 2006). Such responses could lead to genetic specificity in interactions between coinfecting parasites, because unique antigenic variation in each exposure combination can trigger different immune cascades that modify parasite infection success. Possible candidates for such molecular mechanisms include lectin-like receptors and natural antibodies, both of which are present in fish innate immune defense and can differentiate between parasite antigens (reviewed by Magnadóttir 2006). Similarly, the dependence of the interactions on the exposure dose may be related to the strengtt of fish immune responses during parasite invasion. In mammals, for example, parasite exposure dose can even determine the type of immune responses that are elicited (Bancroft et al. 2001; Bleay et al. 2007). Such dose-dependent changes could be very important in determining the interactions among coinfecting parasites. However, to our knowledge, such effects are poorly understood in fist. immune system, the research limiting to studies investigating the protective effect of vaccines (LaPatra et al. 2000; Lorenzen et al. 2000). Nevertheless, also those studies have reported significant dose effects.

To conclude, our results indicate that interactions among coinfecting parasite species can show complex genetic specificity that is further modified by ecological context of the exposure event (i.e., exposure dose). Since the presence as well as the genetic identity of the coinfectin parasites can show high variation among host individuals in nature, such interactions may have important implications for parasite evolution by promoting maintenance of genetic variation. To our knowledge, our study is the first one demonstrating genetic specificity in the interactions among coinfecting parasites. This finding may have wide implications also in many other host-parasite interactions. This is because multiple infections are the rule in nature and variation in other environmental factors ha: been reported to interact with genetic variation in parasite traits across a broad range of different host-parasite systems including animal (Vale and Little 2009), plant (Laine 2007), fungus (Bryner and Rigling 2011), and protist (Fels and Kaltz 2006) hosts. Furthermore, coinfections with multiple parasite species and genotypes are suggested tc $\rightarrow$ play important roles in epidemiology, disease severity, and evolution of parasite virulence (e.g., Lello et al. 2005; de Roode et al. 2005). If genetically specific interactions observed here are common in nature, this can also complicate the predictions considering the role of coinfections in such ecological and evolutionary processes.

\section{Acknowledgments}

We thank S. Reece, B. Sadd, and anonymous reviewers for their helpful comments on the manuscript. Konnevesi Research Station provided the facilities for the experiment. The study was funded by the Emil Aaltonen Foundation (O.S.), the Academy of Finland (A.K.), the Academy of Finland's Centre of Excellence in Evolutionary Research (A.K., C.R., K.-R.L., J.J.), and the Swiss National Science Foundation (J.J.).

\section{Literature Cited}

Aaltonen, T. M., E. I. Jokinen, and E. T. Valtonen. 1994. Antibodysynthesis in roach (Rutilus rutilus): analysis of antibody-secreting cells in lymphoid organs with ELISPOT-assay. Fish and Shellfish Immunology 4:129-140.

Adams, D. B., B. H. Anderson, and R. G. Windon. 1989. Crossimmunity between Haemonchus contortus and Trichostrongylus colubriformis in sheep. International Journal for Parasitology 19:717722 .

Alexander, H. M., J. Antonovics, and A. W. Kelly. 1993. Genotypic variation in plant-disease resistance: physiological resistance in relation to field disease transmission. Journal of Ecology 81:325365.

Bancroft, A. J., K. J. Else, N. E. Humphreys, and R. K. Grencis. 2001. The effect of challenge and trickle Trichuris muris infections on the polarisation of the immune response. International Journal for Parasitology 31:1627-1637.

Blanford, S., M. B. Thomas, C. Pugh, and J. K. Pell. 2003. Temperature checks the Red Queen? resistance and virulence in a fluctuating environment. Ecology Letters 6:2-5.

Bleay, C., C. P. Wilkes, S. Paterson, and M. E. Viney. 2007. Densitydependent immune responses against the gastrointestinal nematode Strongyloides ratti. International Journal for Parasitology 37: 1501-1509.

$\rightarrow$ Brassard, P., M. E. Rau, and M. A. Curtis. 1982. Infection dynamics of Diplostomum spathaceum cercariae and parasite-induced mortality of fish hosts. Parasitology 85:489-493.

$\rightarrow$ Bryner, S. F., and D. Rigling. 2011. Temperature-dependent genotypeby-genotype interaction between a pathogenic fungus and its hyperparasitic virus. American Naturalist 177:65-74.

$\rightarrow$ Carius, H. J., T. J. Little, and D. Ebert. 2001. Genetic variation in a host-parasite association: potential for coevolution and frequencydependent selection. Evolution 55:1136-1145.

Chappell, L. H., L. J. Hardie, and C. J. Secombes. 1994. Diplostomiasis: the disease and host- parasite interactions. Pages 59-86 in A. W. Pike and J. W. Lewis, eds. Parasitic diseases of fish. Samara, Dyfed, Wales.

Cotter, S. C., L. E. Kruuk, and K. Wilson. 2004. Costs of resistance: genetic correlations and potential trade-offs in an insect immune system. Journal of Evolutionary Biology 17:421-429.

$\rightarrow$ de Roode, J. C., R. Pansini, S. J. Cheesman, M. E. H. Helinski, S. Huijben, A. R. Wargo, A. S. Bell, B. H. K. Chan, D. Walliker, and A. F. Read. 2005. Virulence and competitive ability in genetically diverse malaria infections. Proceedings of the National Academy of Sciences of the USA 102:7624-7628. 
$\rightarrow$ Erasmus, D. A. 1959. The migration of Cercaria X Baylis (Strigeida) within the fish intermediate host. Parasitology 49:173-190.

$\rightarrow$ Ezenwa, V. O., R. S. Etienne, G. Luikart, A. Beja-Pereira, and A. E Jolles. 2010. Hidden consequences of living in a wormy world: nematode-induced immune suppression facilitates tuberculosis invasion in African buffalo. American Naturalist 176:613-624.

$\rightarrow$ Fels, D., and O. Kaltz. 2006. Temperature-dependent transmission and latency of Holospora undulata, a micronucleus-specific parasite of the ciliate Paramecium caudatum. Proceedings of the Royal Society B: Biological Sciences 273:1031-1038.

$\rightarrow$ Gillespie, J. H., and M. Turelli. 1989. Genotype-environment interactions and the maintenance of polygenic variation. Genetics 121 129-138.

$\rightarrow$ Grosholz, E. D. 1994. The effects of host genotype and spatial distribution on trematode parasitism in a bivalve population. Evolution 48:1514-1524.

$\rightarrow$ Hedrick, P. W. 2006. Genetic polymorphism in heterogeneous environments: the age of genomics. Annual Review of Ecology and Systematics 37:67-93.

$\rightarrow$ Henter, H. J. 1995. The potential for coevolution in a host-parasitoic system. II. Genetic-variation within a population of wasps in the ability to parasitize an aphid host. Evolution 49:439-445.

Holmes, J. C., and P. W. Price. 1986. Communities of parasites. Pages 187-213 in J. Kikkawa and D. J. Anderson, eds. Community ecol ogy: pattern and process. Blackwell, Melbourne.

$\rightarrow$ Kaltz, O., and J. A. Shykoff. 2002. Within- and among-populatior variation in infectivity, latency and spore production in a hostpathogen system. Journal of Evolutionary Biology 15:850-860.

$\rightarrow$ Karvonen, A., S. Paukku, E. T. Valtonen, and P. J. Hudson. 2003. Transmission, infectivity and survival of Diplostomum spathaceum cercariae. Parasitology 127:217-224.

$\rightarrow$ Karvonen, A., C. Rellstab, K.-R. Louhi, and J. Jokela. 2012. Syn chronous attack is advantageous: mixed genotype infections lead to higher infection success in trematode parasites. Proceedings of the Royal Society B: Biological Sciences 279:171-176.

$\rightarrow$ Karvonen, A., P. Terho, O. Seppälä, J. Jokela, and E. T. Valtonen 2006. Ecological divergence of closely related Diplostomum (Trematoda) parasites. Parasitology 133:229-235.

$\rightarrow$ Koskela, T., S. Puustinen, V. Salonen, and P. Mutikainen. 2002. Resistance and tolerance in a host plant-holoparasitic plant interaction: genetic variation and costs. Evolution 56:899-908.

$\rightarrow$ Koskella, B., T. Giraud, and M. E. Hood. 2006. Pathogen relatedness affects the prevalence of within-host competition. American Naturalist 168:121-126.

$\rightarrow$ Kurtz, J., and K. P. Sauer. 1999. The immunocompetence handicap hypothesis: testing the genetic predictions. Proceedings of the Royal Society B: Biological Sciences 266:2515-2522.

$\rightarrow$ Laine, A.-L. 2007. Pathogen fitness components and genotypes differ in their sensitivity to nutrient and temperature variation in a wila plant-pathogen association. Journal of Evolutionary Biology 20: 2371-2378.

$\rightarrow-$ 2011. Context-dependent effects of induced resistance under co-infection in a plant-pathogen interaction. Evolutionary Applications 4:696-707.

$\rightarrow$ LaPatra, S. E., S. Corbeil, G. R. Jones, W. D. Shewmaker, and G. Kurath. 2000. The dose-dependent effect on protection and humoral response to a DNA vaccine against infectious hematopoietic necrosis (IHN) virus in subyearling rainbow trout. Journal of Aquatic Animal Health 12:181-188.

$\rightarrow$ Lazzaro, B. P., and T. J. Little. 2009. Immunity in a variable world
Philosophical Transactions of the Royal Society B: Biological Sciences 364:15-26.

Lello, J., B. Boag, A. Fenton, I. R. Stevenson, and P. J. Hudson. 2004. Competition and mutualism among the gut helminths of a mammalian host. Nature 428:840-844.

$\rightarrow$ Lello, J., B. Boag, and P. J. Hudson. 2005. The effect of single and concomitant pathogen infections on condition and fecundity of the wild rabbit (Oryctolagus cuniculus). International Journal for Parasitology 35:1509-1515.

$\rightarrow$ Lively, C. M. 1989. Adaptation by a parasitic trematode to local populations of its snail host. Evolution 43:1663-1671.

$\rightarrow$ Lorenzen, E., K. Einer-Jensen, T. Martinussen, S. E. LaPatra, and N. Lorenzen. 2000. DNA vaccination of rainbow trout against viral hemorrhagic septicemia virus: a dose-response and time-course study. Journal of Aquatic Animal Health 12:167-180.

$\rightarrow$ Louhi, K.-R., A. Karvonen, C. Rellstab, and J. Jokela. 2010. Is the population genetic structure of complex life cycle parasites determined by the geographic range of the most motile host? Infection, Genetics and Evolution 10:1271-1277.

Luijckx, P., F. Ben-Ami, L. Mouton, L. Du Pasquier, and D. Ebert. 2011. Cloning of the unculturable parasite Pasteuria ramosa and its Daphnia host reveals extreme genotype-genotype interactions. Ecology Letters 14:125-131.

Magnadóttir, B. 2006. Innate immunity of fish (overview). Fish and Shellfish Immunology 20:137-151.

Mitchell, S. E., E. S. Rogers, T. J. Little, and A. F. Read. 2005. Hostparasite and genotype-by-environment interactions: temperature modifies potential for selection by a sterilizing pathogen. Evolution 59:70-80.

Patrick, M. J. 1991. Distribution of enteric helminths in Glaucomys volans L. (Sciuridae): a test for competition. Ecology 72:755-758.

$\rightarrow$ Price, J. S., J. D. Bever, and K. Clay. 2004. Genotype, environment, and genotype by environment interactions determine quantitative resistance to leaf rust (Coleosporium asterum) in Euthamia graminifolia (Asteraceae). New Phytologist 162:729-743.

Råberg, L., J. C. de Roode, A. S. Bell, P. Stamou, D. Gray, and A. F. Read. 2006. The role of immune-mediated apparent competition in genetically diverse malaria infections. American Naturalist 168: 41-53.

$\rightarrow$ Rauch, G., M. Kalbe, and T. B. H. Reusch. 2005. How a complex life cycle can improve a parasite's sex life. Journal of Evolutionary Biology 18:1069-1075.

$\rightarrow-$ 2006. One day is enough: rapid and specific host-parasite interaction in a stickleback-trematode system. Biology Letters 2: $382-384$.

$\rightarrow$ - 2008. Partitioning average competition and extreme-genotype effects in genetically diverse infections. Oikos 117:399-405.

Read, A. F., and L. H. Taylor. 2001. The ecology of genetically diverse infections. Science 292:1099-1102.

$\rightarrow$ Rellstab, C., K.-R. Louhi, A. Karvonen, and J. Jokela. 2011. Analysis of trematode parasite communities in fish eye lenses by pyrosequencing of naturally pooled DNA. Infection, Genetics and Evolution 11:1276-1286.

$\rightarrow$ Reusch, T. B. H., G. Rauch, and M. Kalbe. 2004. Polymorphic microsatellite loci for the trematode Diplostomum pseudospathaceum. Molecular Ecology Notes 4:577-579.

Roff, D. A. 1997. Evolutionary quantitative genetics. Chapman \& Hall, New York.

$\rightarrow$ Salvaudon, L., V. Héraudet, and J. Shykoff. 2005. Parasite-host fitness 
trade-offs change with parasite identity: genotype-specific inter $\rightarrow$ Staves, P. A., and R. J. Knell. 2010. Virulence and competitiveness: actions in a plant-pathogen system. Evolution 59:2518-2524. testing the relationship during inter- and intraspecific mixed inSchlichting, C. D., and M. Pigliucci. 1998. Phenotypic evolution: a reaction norm perspective. Sinauer, Sunderland, MA. fections. Evolution 64:2643-2652.

$\rightarrow$ Schmid-Hempel, P., K. Puhr, N. Krüger, C. Reber, and R. SchmidHempel. 1999. Dynamics and genetic consequences of variation in horizontal transmission for a microparasitic infection. Evolution 53:426-475.

$\rightarrow$ Selva, L., D. Viana, G. Regev-Yochay, K. Trzcinski, J. M. Corpa, I. Lasa, R. P. Novick, and J. R. Penades. 2009. Killing niche com petitors by remote-control bacteriophage induction. Proceedings of the National Academy of Sciences of the USA 106:1234-1238.

$\rightarrow$ Seppälä, O., and J. Jokela. 2010. Maintenance of genetic variation in immune defense of a freshwater snail: role of environmental heterogeneity. Evolution 64:2397-2407.

$\rightarrow$ Seppälä, O., A. Karvonen, and E. T. Valtonen. 2007. Phenotypic variation in infectivity of Diplostomum spathaceum cercariae withirı a population. Journal of Parasitology 93:1244-1246.

$\rightarrow$ Seppälä, O., A. Karvonen, E. T. Valtonen, and J. Jokela. 2009. In teractions among co-infecting parasite species: a mechanism maintaining genetic variation in parasites? Proceedings of the Royal Society B: Biological Sciences 276:691-697.

$\rightarrow$ Taylor, L. H., D. Walliker, and A. F. Read. 1997. Mixed-genotype infections of malaria parasites: within-host dynamics and transmission success of competing clones. Proceedings of the Royal Society B: Biological Sciences 264:927-935.

$\rightarrow$ Vale, P. F., and T. J. Little. 2009. Measuring parasite fitness under genetic and thermal variation. Heredity 103:102-109.

$\rightarrow$ Valtonen, E. T., and D. I. Gibson. 1997. Aspects of the biology of diplostomid metacercarial (Digenea) populations occurring in fishes in different localities of northern Finland. Annales Zoologici Fennici 34:47-59.

$\rightarrow$ Valtonen, E. T., K. Pulkkinen, R. Poulin, and M. Julkunen. 2001. The structure of parasite component communities in brackish water fishes of the northeastern Baltic Sea. Parasitology 122:471-481.

$\rightarrow$ Voutilainen, A., H. Huuskonen, and J. Taskinen. 2010. Penetration and migration success of Diplostomum spp. cercariae in arctic char. Journal of Parasitology 96:232-235.

$\rightarrow$ Whyte, S. K., C. J. Secombes, and L. H. Chappell. 1991. Studies on the infectivity of Diplostomum spathaceum in rainbow trout (Oncorhynchus mykiss). Journal of Helminthology 65:169-178.

$\rightarrow$ Seppälä, O., K. Liljeroos, A. Karvonen, and J. Jokela. 2008. Host condition as a constraint for parasite reproduction. Oikos 117: 749-753.

$\rightarrow$ Williams, M. O. 1966. Studies on the morphology and life-cycle of Diplostomum (Diplostomum) gasterostei (Strigeida: Trematoda). Parasitology 56:693-706.

$\rightarrow$ Stables, J. N., and L. H. Chappell. 1986. Diplostomum spathaceum (Rud. 1819): effects of physical factors on the infection of rainbow trout (Salmo gairdneri) by cercariae. Parasitology 93:71-79.
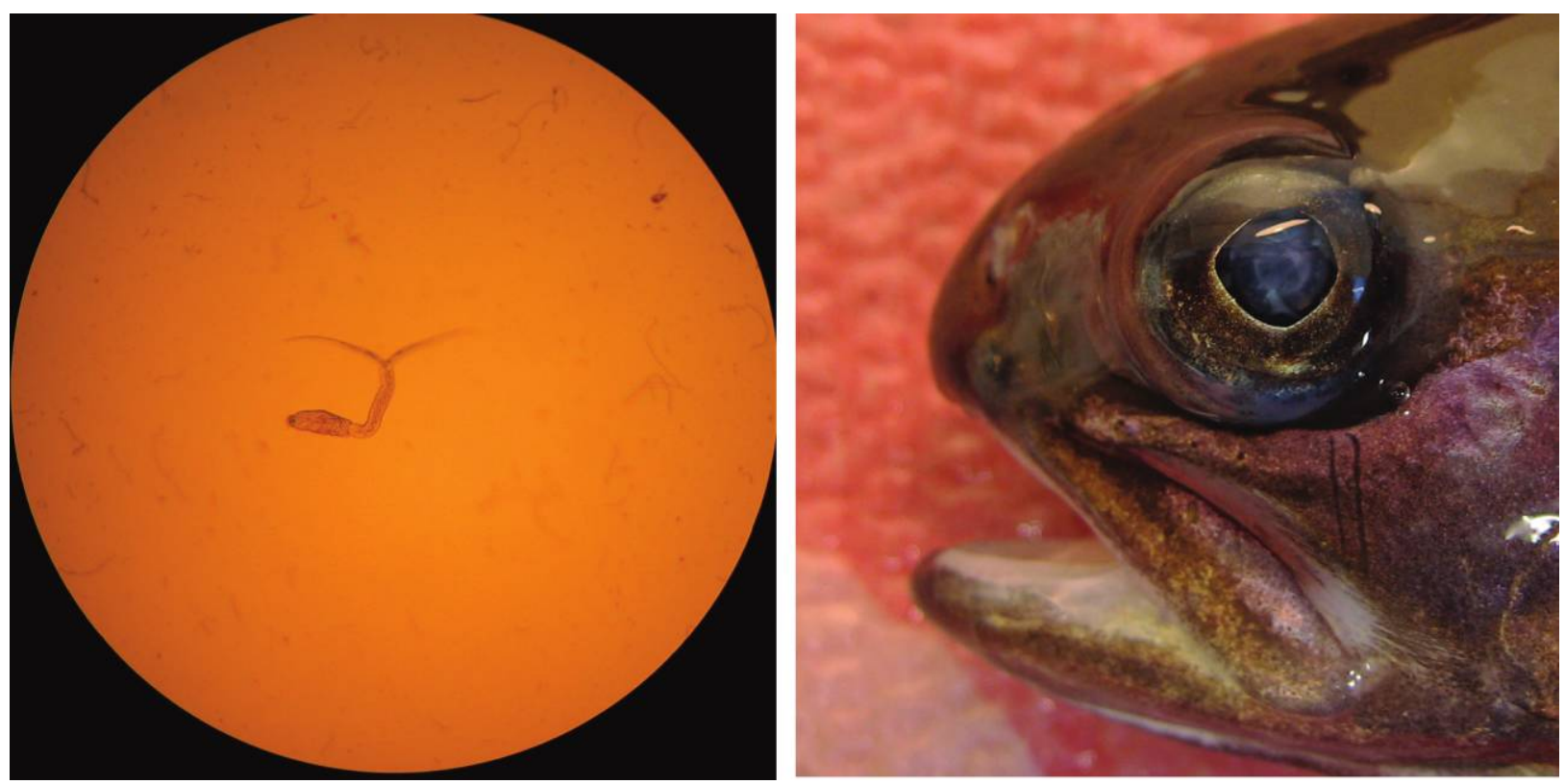

Left, Diplostomum pseudospathaceum cercaria parasite. Photograph by Christian Rellstab. Right, rainbow trout infected with eye fluke. Photograph by Anssi Karvonen. 